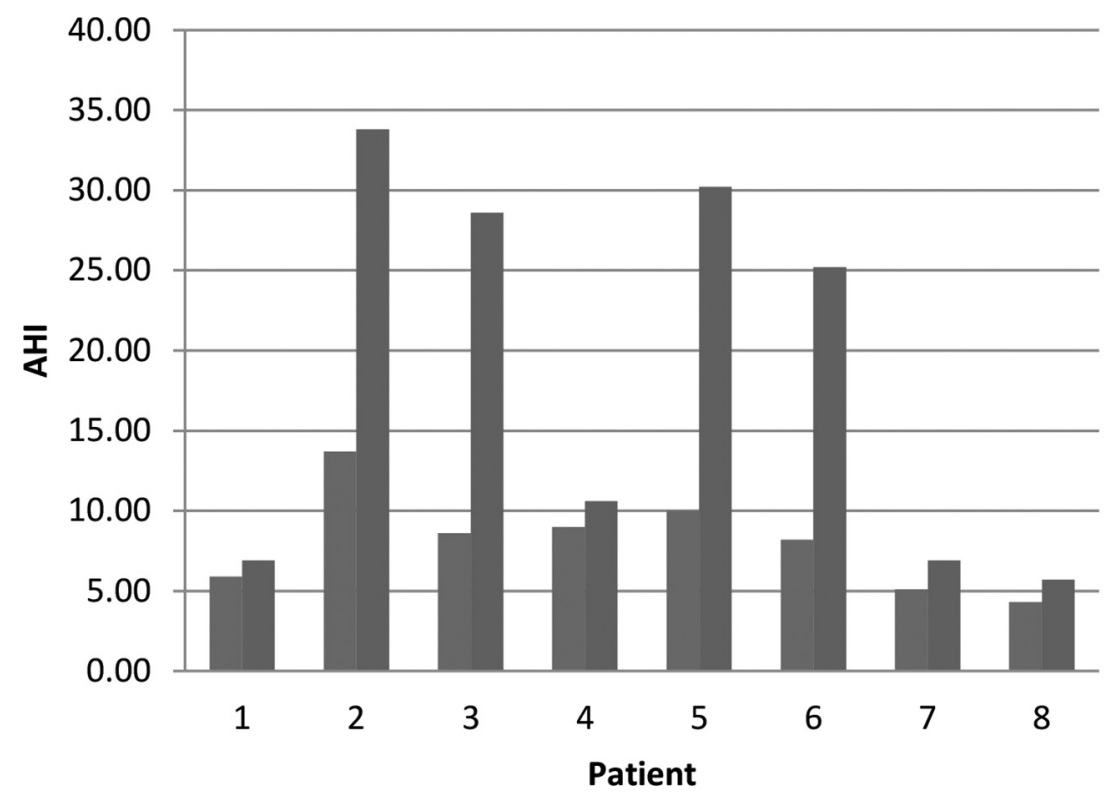

Nox T3 AHI

NOX A1 AHI

Abstract P018 Figure 1 A Graph to show the AHI's gathered from a Nox T3 and a Nox A1

Abstract P018 Table 1
\begin{tabular}{|l|c|}
\hline $\mathrm{n}=8$ & Mean $(\mathrm{sd})$ \\
\hline Sex $(\mathrm{m})$ & 6 \\
\hline Age (years) & $38(12)$ \\
\hline BMI (kg/m²) & $32.1(7.1)$ \\
\hline Collar Size (inches) & $16.6(2.1)$ \\
\hline Epworth Score & $13(2)$ \\
\hline Sleep Latency (min) & 18.2 \\
& $(23.4)$ \\
\hline PLMS Index & 31.5 \\
& $(38.7)$ \\
\hline Sp02 T3 (\%) & $94(2)$ \\
\hline ODI T3 (/hr) & $7.1(3.6)$ \\
\hline AHI T3 (/hr) & $8.1(3)$ \\
\hline Sp02 A1 (\%) & $92(4)$ \\
\hline ODI A1 (/hr) & 17.7 \\
& $(12.1)$ \\
\hline AHI A1 (/hr) & $18.4(12)$ \\
\hline
\end{tabular}

symptomatic mild OSAS patients. Reasons may include location of sleep and clinical support with sleep study setup. In this patient group it may be advised that PSG is required in order to confirm a diagnosis of OSAS and severity in order to select the most appropriate treatment modality and optimisation of treatment selections. Larger multicentre studies are required to substantiate the results from this study.

\section{P019 SURFACE CRANIOFACIAL PHENOTYPE AND PREDICTION MODELS FOR OBSTRUCTIVE SLEEP APNOEA: A 3-D EVALUATION}

1,2Bahn Agha*, 'Lifong Zou, 'Ama Johal. 'Queen Mary University of London, The London School of Medicine and Dentistry, London, UK; ${ }^{2}$ Mustansiriyah University, College of Dentistry, Baghdad, IRAQ

\subsection{6/bmjresp-2019-bssconf.19}

Aims Obstructive sleep apnoea (OSA) is considered as a major healthcare problem, remaining relatively underdiagnosed and associated with significant comorbidity (Rejón-Parrilla et al., 2014). The present study aims to explore: the existence of a craniofacial phenotype in adults with OSA, the ability to predict the condition from clinical and surface craniofacial structures and the presence of a surface facial marker for OSA.

Methods A case-control study was conducted with 118 middle-aged Caucasian males (56 controls; 62 OSA). Each undergoing a clinical examination including body mass index,

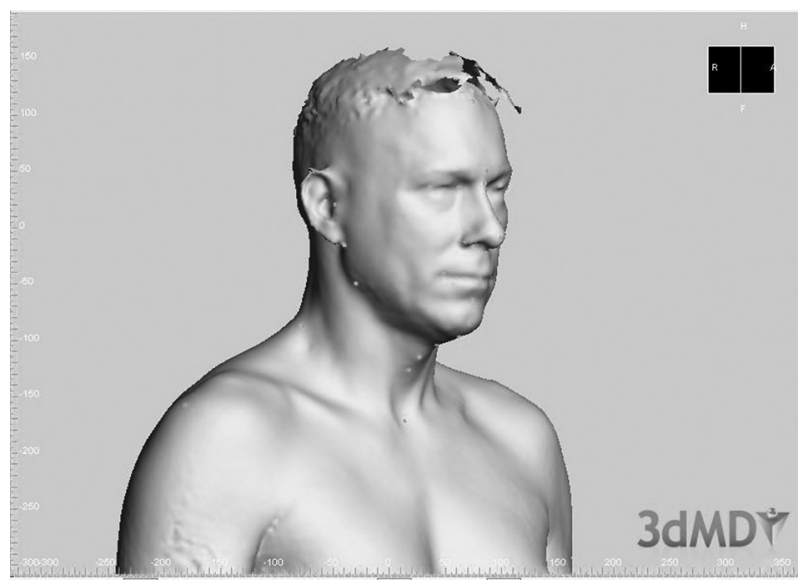

Abstract P019 Figure 1 3-D Sterophotogrammetry shows surface surface landmarks of the face, neck an upper torso 

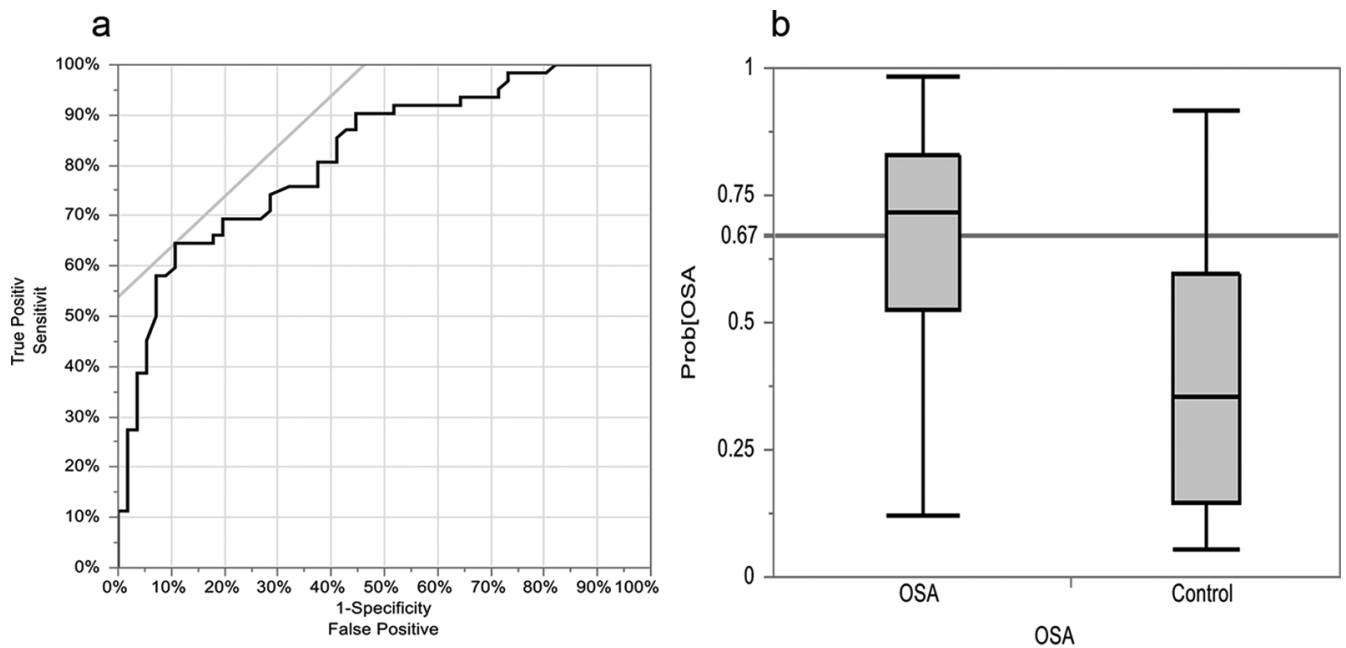

C
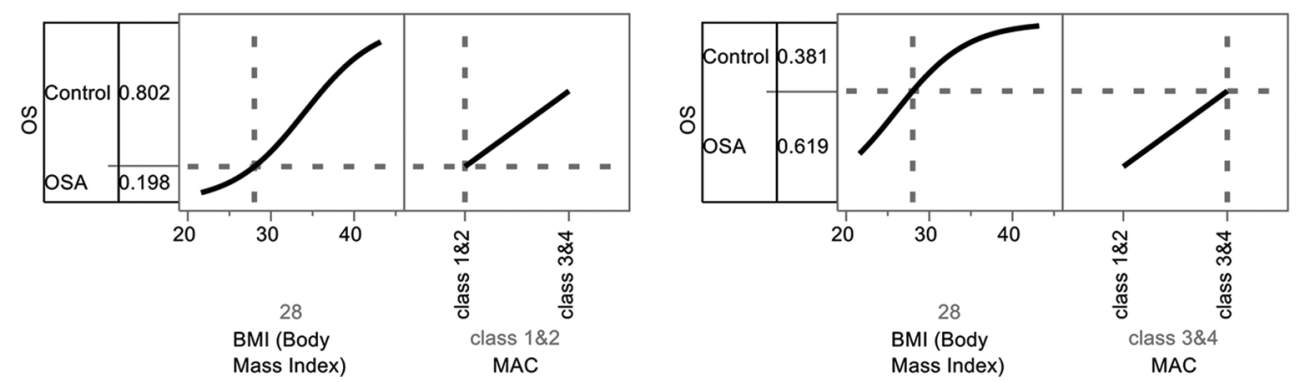

Abstract P019 Figure 2 a) Receiver operating characteristic (ROC) curve for backward logistic regression model for surface and clinical variables with area under the curve (AUC) of 0.82 . b) Box plot showing logistic regression model score for OSA subjects and controls. A cut-off value of 0.67 (red line, b) produced $65 \%$ sensitivity and $89 \%$ specificity for identification of OSA patients with an AHI $\geq 5$ events/hour. c) Prediction profilers for logistics multiple regression - surface and clinical predictors (backward model). Prediction formula: Prob (OSA) $=1 /(1+$ Exp $(-((-7.88)+0.23 * B M I+$ $1.88 * \mathrm{MAC}))$ )

Mallampati airway classification, sleep apnoea clinical score, Epworth sleepiness scale and 3-D stereophotography for surface craniofacial analysis (figure1).

Results Surface craniofacial risk factors (phenotype) were identified for OSA Caucasian males, with the predominant characteristics being: an enlarged neck circumference $(\mathrm{p}<0.001)$, short neck $(\mathrm{p}<0.001)$, large mandibular width $(\mathrm{p}<0.001)$, forward head posture $(\mathrm{p}<0.001)$ and increased lower anterior facial height $(\mathrm{P}<0.002)$. Multiple regression analysis for the surface predictors, both with and without clinical variables, identified a range of prediction models with moderate to high sensitivity and specificity, with an area under the receiver operating characteristics curve (AUC) between 0.73-0.82. A higher positive and lower negative likelihood-ratios were identified for the combination model of the surface and clinical variables $(L R+6.02, L R-0.40$ respectively) when compared to the surface model alone. A high positive post-test probability and odds ratio $(87 \%$, $\mathrm{OR}=6.8$, respectively) were also identified in the combination model. The surface model, with and without clinical variables, not only successfully identified OSA subjects from controls (AUC $=0.77,0.82$ respectively) but also presented as a marker (figure 2).

Conclusion This case-control study demonstrated the existence of a surface craniofacial phenotypic pattern, identified a predictive model and marker for OSA in Caucasian males, using 3D-surface imaging analysis and clinical tools.

\section{P020 AN AUDIT OF SLEEP STUDY RESULTS IN PATIENTS UNDERGOING PRE-OPERATIVE ASSESSMENT}

Vicky Cooper*. Salford Royal NHS Foundation Trust, Salford

10.1136/bmjresp-2019-bssconf.20

Introduction Undiagnosed obstructive sleep apnoea (OSA) presents a significant risk to patients undergoing general anaesthesia. The STOP-BANG questionnaire is a useful screening tool for predicting a high probability of OSA. This study examines the results of the pre-operative screening at Salford Royal NHS Foundation Trust.

Methods The sleep study results from 857 patients (658 male) aged 20-87 years $(57.1 \pm 0.4$ years) who underwent a preoperative assessment and scored $\geq 3$ on the STOP-BANG questionnaire were retrospectively analysed. Patients were categorised as have no, mild, moderate or severe OSA according to their Apnoea Hypopnoea Index (AHI) $(n=675)$ or Oxygen Desaturation Index (ODI) $(n=182)$. using AASM criteria b (4\% desaturation).

The effects of gender, age, body mass index (BMI), neck circumference (NC) and Epworth Sleepiness Score (ESS) were independently assessed and differences between groups were compared using One Way ANOVA. Results are reported as mean \pm standard error.

Results The number of patients having no, mild, moderate or severe OSA were 215 (25\%), 250 (29\%), 214 (25\%) and 178 (21\%), respectively. 